\title{
Does statin use have a disease modifying effect in symptomatic knee osteoarthritis? Study protocol for a randomised controlled trial
}

Yuanyuan Wang ${ }^{1 *}$, Andrew Tonkin ${ }^{1}$, Graeme Jones$^{2}$, Catherine Hill ${ }^{3,4}$, Changhai Ding ${ }^{1,2}$, Anita E. Wluka', Andrew Forbes ${ }^{1}$ and Flavia M. Cicuttini ${ }^{1}$

\begin{abstract}
Background: Osteoarthritis $(\mathrm{OA})$ is a major clinical and public health problem, with no current medications approved as having disease modifying effects. HMG-CoA reductase inhibitors, or "statins", a drug class widely used to prevent cardiovascular events, could potentially affect OA progression via a number of mechanisms including their effects on lipid metabolism and inflammation. The aim of this multicentre, randomised, double-blind, placebo-controlled trial is to determine whether atorvastatin reduces the progression of knee structural changes and symptoms over 2 years in patients with symptomatic knee OA.
\end{abstract}

Methods/design: 350 patients with symptomatic knee OA will be recruited through the OA Clinical Trial Network (in Melbourne, Hobart and Adelaide). They will be randomly allocated to the two arms of the study, receiving either $40 \mathrm{mg}$ of atorvastatin or identical placebo once daily for 2 years. Magnetic resonance imaging of the knee will be performed at baseline and 2 years later. Knee structure, symptoms and function will be assessed using validated methods. The primary outcome is annual percentage change in knee cartilage volume. Secondary outcomes include progression of cartilage defects, bone marrow lesions, knee pain and function. The primary analysis will be by intention to treat, but per protocol analyses will also be performed.

Discussion: The study will provide high-quality evidence to address whether atorvastatin has a novel disease modifying effect in OA by delaying the structural and symptomatic progression of knee OA. Thus, the trial has major public health and clinical importance, as if found to be beneficial, atorvastatin could produce substantial cost savings by delaying and possibly reducing the need for joint replacement surgery, and provide marked improvements in quality of life for people with OA.

Trial registration: Australian New Zealand Clinical Trials Registry: ACTRN12613000190707, registered on 18 February 2013.

Keywords: Statins, Osteoarthritis, Cartilage, Pain, Magnetic resonance imaging

\footnotetext{
* Correspondence: yuanyuan.wang@monash.edu

${ }^{1}$ Department of Epidemiology and Preventive Medicine, Monash University,

Alfred Hospital, Melbourne 3004, Australia

Full list of author information is available at the end of the article
} 


\section{Background}

Osteoarthritis (OA), characterised by progressive structural deterioration and symptoms, is a significant clinical and public health problem, causing pain, disability and a substantial health care burden. It is a major contributor to the burden of disease, being ranked as the eleventh highest contributor of global disability among 291 conditions in the Global Burden of Disease study and associated with 71.1 million years lived with disability, an increase of $64 \%$ from 1990 to 2010 [1]. Symptomatic knee OA makes a significant impact on society, with $44.7 \%$ of people developing this condition in their lifetime [2]. Current treatments for OA mainly focus on the improvement of symptoms. Although several drugs and nutraceuticals have been examined for their effects on slowing structural progression of OA over the past 10 years, none has been approved as a disease modifying OA drug [3]. Many patients with symptomatic end-stage OA are eventually faced with joint replacement as the only treatment option to improve quality of life, and the number of joint replacements has continued to increase worldwide.

There is growing evidence that $\mathrm{OA}$ is a multifactorial complex disorder, with a significant metabolic component in its pathogenesis, in which various interrelated lipid, metabolic and inflammatory mediators contribute to the initiation and progression of the disease [4-6]. These metabolic factors appear to be more important in OA of the knee than the hip [7].

In the Chingford study of 1,003 middle-aged women, hypercholesterolaemia was associated with increased risk of knee OA independent of obesity, and the association was stronger for bilateral knee OA than for unilateral knee OA [8]. In the Ulm study of 809 patients with joint replacement due to $\mathrm{OA}$, high serum cholesterol levels were independently associated with increased risk of knee OA [9]. In recent longitudinal magnetic resonance imaging (MRI) studies, we showed that higher serum cholesterol and triglyceride levels were associated with adverse structural changes (increased incidence of bone marrow lesions, BMLs) in asymptomatic middle-aged women over 2 years [10], while higher serum levels of high-density lipoprotein (HDL) cholesterol were protective against worsening of BMLs in older adults over 2.7 years [11]. BMLs are early knee structural changes associated with knee pain and cartilage loss [12, 13] and joint replacement in OA [14].

Inflammation has also been implicated in the pathogenesis of OA [6]. In human OA, the histological changes include increased vascularity, lining layer thickening, and inflammatory cell infiltration with increased numbers of lining cells. A mixed population of inflammatory cells (macrophages, activated $\mathrm{T}$ cells) is seen in the sublining tissue [15]. These cells, as well as chondrocytes, produce pro-inflammatory cytokines, such as interleukin (IL)-1 $\beta$,
IL-6, and tumour necrosis factor (TNF)- $\alpha$ [15], which are detectable even in early OA [15]. Synovitis identified by arthroscopy predicted increased cartilage loss over 1 year [16], and synovitis detected by contrast-enhanced MRI was associated with increased cartilage loss over 30 months [17]. Circulating levels of C-reactive protein were associated with disease progression and decreased cartilage volume at the knee $[18,19]$. Increased serum level of IL-6 was a significant predictor of radiographic OA 5-10 years later in women [20], associated with increased knee cartilage loss over 2 years in OA [21] and increased cartilage volume loss over 3 years in older adults [22]. We found that elevated TNF- $\alpha$ levels were also associated with increased rate of cartilage volume loss over 3 years in older adults [22].

Hydroxy-methyl-glutaryl coenzyme A (HMG-CoA) reductase inhibitors (statins), a drug class used to lower low-density lipoprotein (LDL) cholesterol levels with smaller effects on lowering triglycerides and increasing HDL cholesterol levels, are proven and have been widely used to prevent cardiovascular events [23]. In addition to lowering LDL cholesterol, statins have a broad range of pleiotropic effects, including anti-inflammatory effects, which could exert an effect on synovium and cartilage [24]. Data from observational studies of statins are somewhat conflicting (Table 1). Some large cohort studies have shown that statin use is associated with reduced incidence and progression of OA $[25,26]$ and that higher statin dose and larger statin increments are associated with reduced incident episode of clinically defined OA [27]. However, other cohort studies have reported that statin use is associated with an increased incidence of OA and arthropathy [28, 29], or that statin use is not associated with improvement in knee pain, function, or structural progression of knee OA [30]. Thus, the current evidence for the association between statin use and the risk of OA comes from observational human studies which are subject to bias and confounding, and the overall data are inconclusive. No randomised controlled trials of statins have been performed so far.

We will conduct a randomised controlled trial to determine whether atorvastatin has a disease modifying effect in OA by reducing the MRI-assessed structural progression (knee cartilage volume loss, progression of cartilage defects and BMLs) and symptoms in patients with symptomatic knee OA over 2 years. It was hypothesised that atorvastatin use will reduce the rate of knee cartilage volume loss (primary hypothesis), the progression of cartilage defects and BMLs (secondary hypothesis), and improve symptoms and function (secondary hypothesis) over 2 years compared with placebo in people with symptomatic knee OA. If statins are proven to be effective, they will offer a novel therapeutic approach to reducing the progression of knee OA. 
Table 1 Summary of findings from human epidemiological studies for association between statin use and risk of osteoarthritis

\begin{tabular}{|c|c|c|c|c|}
\hline Author, year & Study design & Participants & Outcome measure & Main results \\
\hline $\begin{array}{l}\text { Beattie MS, et al. } \\
2005 \text { [29] }\end{array}$ & $\begin{array}{l}\text { Prospective cohort } \\
\text { study }\end{array}$ & $\begin{array}{l}5,674 \text { elderly women aged } \\
\geq 65 \text { years from the Study of } \\
\text { Osteoporotic Fractures }\end{array}$ & Radiographic hip OA & $\begin{array}{l}\text { Statin use was associated with } \\
\text { an increased risk of developing } \\
\text { radiographic hip OA, but did not } \\
\text { adversely affect the progression } \\
\text { of established disease. }\end{array}$ \\
\hline $\begin{array}{l}\text { Chodick G, et al. } \\
2010 \text { [26] }\end{array}$ & $\begin{array}{l}\text { Retrospective } \\
\text { population-based } \\
\text { cohort study }\end{array}$ & $\begin{array}{l}193,770 \text { individuals from } \\
\text { the computerised medical } \\
\text { databases of a large health } \\
\text { organisation }\end{array}$ & $\begin{array}{l}\text { International Classification of } \\
\text { Diseases, 9th revision, diagnosis } \\
\text { codes }\end{array}$ & $\begin{array}{l}\text { Persistent statin use was associated } \\
\text { with a reduced incidence of OA. }\end{array}$ \\
\hline $\begin{array}{l}\text { Clockaerts S, } \\
\text { et al. } 2012 \text { [25] }\end{array}$ & $\begin{array}{l}\text { Prospective } \\
\text { population-based } \\
\text { cohort study }\end{array}$ & $\begin{array}{l}2,921 \text { participants aged } \geq 55 \\
\text { years in the Rotterdam study }\end{array}$ & Radiographic knee and hip OA & $\begin{array}{l}\text { Statin use was associated with } \\
\text { reduced incidence and progression } \\
\text { of radiographic knee OA, but not } \\
\text { radiographic hip OA over } 6.5 \text { years. }\end{array}$ \\
\hline $\begin{array}{l}\text { Kadam UT, et al. } \\
2013 \text { [27] }\end{array}$ & $\begin{array}{l}\text { Retrospective } \\
\text { cohort study }\end{array}$ & $\begin{array}{l}16,609 \text { adults aged } \geq 40 \text { years } \\
\text { from the UK General Practice } \\
\text { Research Database }\end{array}$ & $\begin{array}{l}\text { Clinically defined OA: OA-related } \\
\text { diagnostic categories from a } \\
\text { standard clinical classification, } \\
\text { recorded by GPs in the actual } \\
\text { consultations }\end{array}$ & $\begin{array}{l}\text { Higher statin dose and larger statin } \\
\text { increments were associated with } \\
\text { reduced incident episode of clinically } \\
\text { defined OA over 2-10 years. }\end{array}$ \\
\hline $\begin{array}{l}\text { Mansi IA, et al. } \\
2013 \text { [28] }\end{array}$ & $\begin{array}{l}\text { Retrospective } \\
\text { cohort study }\end{array}$ & $\begin{array}{l}\text { 92,360 patients in the San Antonio } \\
\text { Military Multi-Service Market }\end{array}$ & $\begin{array}{l}\text { International Classification of } \\
\text { Diseases, 9th edition, diagnosis } \\
\text { codes }\end{array}$ & $\begin{array}{l}\text { Statin use was associated with an } \\
\text { increased incidence of OA and } \\
\text { arthropathy over } 4 \text { years. }\end{array}$ \\
\hline $\begin{array}{l}\text { Riddle DL, et al. } \\
2013 \text { [30] }\end{array}$ & $\begin{array}{l}\text { Prospective cohort } \\
\text { study }\end{array}$ & $\begin{array}{l}\text { 2,207 participants with } \\
\text { radiographically suspected } \\
\text { or confirmed knee OA in the } \\
\text { Osteoarthritis Initiative }\end{array}$ & $\begin{array}{l}\text { Knee pain, function, and } \\
\text { radiographic knee OA }\end{array}$ & $\begin{array}{l}\text { Statin use was not associated with } \\
\text { improvement in knee pain, function, } \\
\text { or structural progression of knee OA } \\
\text { over } 4 \text { years. }\end{array}$ \\
\hline
\end{tabular}

\section{Methods/design}

\section{Study design}

The Osteoarthritis of the Knee Statin (OAKS) study is a multicentre, randomised, double-blind, placebo-controlled trial over 2 years. The trial was registered at the Australian New Zealand Clinical Trials Registry prior to recruitment, and trial reporting will be guided by the CONSORT Statement [31]. A total of 350 patients with symptomatic knee OA will be recruited in equal numbers via the OA Clinical Trial Network in Melbourne, Hobart and Adelaide, using a combined strategy including collaboration with general practitioners, rheumatologists, and orthopaedic surgeons, as well as advertising through local media. Ethics approval has been obtained from The Alfred Hospital Ethics Committee (521/12), Monash University Human Research Ethics Committee (CF13/595 - 2013000236), Tasmania Health and Medical Human Research Ethics Committee (H0012971), and Human Research Ethics Committee (TQEH/LMH/MH) (HREC/ 13/TQEHLMH/20). Written informed consent will be obtained from all participants.

\section{Inclusion criteria}

The inclusion criteria will be: males and females with symptomatic knee OA for at least 6 months with a pain score of at least $20 \mathrm{~mm}$ on a $100-\mathrm{mm}$ visual analogue scale (VAS); age 40-70 years old; and meeting the American College of Rheumatology (ACR) criteria for symptomatic knee OA [32], assessed by a rheumatologist.

\section{Exclusion criteria}

Participants with any of the following conditions will be excluded: inability to give informed consent; severe radiographic knee OA (grade 3 according to Altman's atlas [33]) or severe knee pain (on standing $>80 \mathrm{~mm}$ on a 100-mm VAS); rheumatoid arthritis or other inflammatory arthritis; significant knee injury; familial hypercholesterolaemia for which statins are indicated, known atherosclerotic cardiovascular disease, diabetes mellitus, taking current lipid lowering therapy, or with previous adverse reaction to statins; absolute cardiovascular risk estimated using the Framingham Risk Equation of more than $15 \%$ within the next 5 years (National Heart Foundation of Australia, 2005); fasting total cholesterol level $>7.5 \mathrm{mmol} / \mathrm{L}$; clinically significant renal disease or abnormal liver function assessed by aspartate aminotransferase and alanine aminotransferase, creatine kinase more than twice the upper limit of laboratory normal range; patients undergoing arthroscopy or open surgery in the index knee in the last 12 months; receiving intra-articular therapy in the index knee in the last 12 months; concomitant use of potent analgesics including opiates; co-morbidity that may limit participation (such as planned index knee joint replacement or medical conditions, for example, malignancy in the past 5 years other than non-melanoma skin cancer); relocation; any 
contraindication to MRI scanning (for example, implanted pacemaker, metal sutures, presence of shrapnel or iron filings in the eye, or claustrophobia); pregnancy, breast feeding, or women trying to become pregnant.

\section{Randomisation and blinding}

Allocation of participants in a 1:1 ratio to either the intervention or control group will be based on computergenerated random numbers prepared by a statistician with no involvement in the trial. Block randomisation will be performed using a central automated allocation procedure, stratified according to the study site. The randomised controlled trial will be a double-blind one, with both participants and investigators assessing outcomes blinded to treatment allocation. Allocation concealment and double blinding will be ensured by 1) the medications being dispensed by the hospital clinical trial pharmacy in each site; 2) the use of an identical placebo tablet; 3 ) objective measures of knee structural changes being made by trained observers blinded to group allocation and the time sequence of MRI scans; 4) subjective measures being taken by research assistants blinded to group allocation.

\section{Intervention}

All participants will be provided usual care by their treating health practitioners. Participants in the intervention arm will receive $40 \mathrm{mg}$ of atorvastatin (Sandoz) once daily, and those in the control arm will receive $40 \mathrm{mg}$ of inactive matching placebo (Pharmaceutical Packaging Professionals Pty Ltd, Thebarton, South
Australia) once daily. Atorvastatin was chosen as it is the most widely prescribed statin and has potent LDL cholesterol lowering effects.

\section{Study procedure}

At screening, participants will complete questionnaires, have a knee X-ray, and undergo biochemical testing including liver function tests, creatine kinase and renal function tests, to ensure inclusion criteria are met. The study knee will be defined as the one with symptomatic $\mathrm{OA}$; if both are symptomatic, the one with least severe radiographic OA (joint space narrowing) will be studied, to reduce loss to follow-up for joint replacement. After screening, study visits will be scheduled for 0 (baseline), 6, 12 and 24 months (Table 2). The same researchers, who are blinded to treatment allocation, will measure all clinical variables, administer questionnaires, monitor compliance, and record adverse events at these visits. Compliance by trial medication will be assessed by pill count. Biochemical testing will be performed at 4 weeks, and at 6,12 and 24 months. At baseline and 24 months the index knee will be imaged on the same whole-body MRI unit.

\section{Quality assurance}

To ensure high-quality execution of the trial in accordance with the protocol, all trial staff will be trained by the chief investigators and provided a standard protocol book (with details of standard operating procedures used for all

Table 2 Timetable and measures to be made

\begin{tabular}{|c|c|c|c|c|c|c|c|}
\hline & Screening & Month 0 (baseline) & Week 4 & Month 6 & Month 12 & Month 18 & Month 24 \\
\hline$\overline{X \text {-ray }}$ & $\sqrt{ }$ & & & & & & \\
\hline Biochemical testing & $\sqrt{ }$ & & $\sqrt{ }$ & $\sqrt{ }$ & $\sqrt{ }$ & & $\sqrt{ }$ \\
\hline Questionnaires & $\sqrt{ }$ & $\sqrt{ }$ & & $\sqrt{ }$ & $\sqrt{ }$ & & $\sqrt{ }$ \\
\hline Knee VAS & $\sqrt{ }$ & $\sqrt{ }$ & & $\sqrt{ }$ & $\sqrt{ }$ & & $\sqrt{ }$ \\
\hline Knee WOMAC & & $\sqrt{ }$ & & $\sqrt{ }$ & $\sqrt{ }$ & & $\sqrt{ }$ \\
\hline IPAQ & & $\sqrt{ }$ & & & & & $\sqrt{ }$ \\
\hline SF-36 & & $\sqrt{ }$ & & & & & $\sqrt{ }$ \\
\hline Concomitant medications & $\sqrt{ }$ & $\sqrt{ }$ & & $\sqrt{ }$ & $\sqrt{ }$ & & $\sqrt{ }$ \\
\hline Smoking & & $\sqrt{ }$ & & & & & v \\
\hline History of joint disease & $\sqrt{ }$ & $\sqrt{ }$ & & & & & $\sqrt{ }$ \\
\hline Co-morbidities & $\sqrt{ }$ & $\sqrt{ }$ & & & & & $\sqrt{ }$ \\
\hline Education and occupation & & $\sqrt{ }$ & & & & & \\
\hline Physical examination & & $\checkmark$ & & & & & $\sqrt{ }$ \\
\hline Height and weight & & $\sqrt{ }$ & & & & & $\sqrt{ }$ \\
\hline Lower limb muscle strength & & $\sqrt{ }$ & & & $\sqrt{ }$ & & $\sqrt{ }$ \\
\hline Knee MRI & & $\sqrt{ }$ & & & & & $\sqrt{ }$ \\
\hline Compliance and adverse events & & & & $\sqrt{ }$ & $\sqrt{ }$ & $\sqrt{ }$ & $\sqrt{ }$ \\
\hline Medication dispensing & & $\sqrt{ }$ & & v & $\sqrt{ }$ & $\sqrt{ }$ & \\
\hline
\end{tabular}


trial contacts, visits, measurements, and monitoring) and case report forms.

\section{Outcome measures \\ Knee MRI}

Knee MRI acquisition at the three study sites is presented in Table 3, including details of sequences and parameters being used.

\section{Primary outcome measure: annual percentage change in tibial cartilage volume}

From sagittal T1-weighted images, medial and lateral tibial cartilage volume will be isolated by manually drawing disarticulation contours around the cartilage boundaries on a section-by-section basis. Measurement error and bias will be reduced by ensuring that one reader measures participants' paired set of images blinded to time sequence, with a second reader performing independent consistency checks. In our previous study, we demonstrated a coefficient of variation of $2.0-3.4 \%$ for this method [34]. We and others have shown that change in tibial cartilage volume over 2 years can be measured using this method and is both statistically and clinically significant in patients with symptomatic knee OA [34-36]. The annual percentage change in tibial cartilage volume will be calculated as (follow-up cartilage volume - baseline cartilage volume)/ baseline cartilage volume/time between MRI scans" 100 .

\section{Secondary outcome measures}

Progression of cartilage defects Cartilage defects will be graded at tibial and femoral sites (0-4) from sagittal images [37]. If multiple cartilage defects are present at one site, the highest grade will be recorded. Our intra- and inter-observer reliability for this method is $0.89-0.94$ and $0.85-0.93$, respectively [37]. The progression of cartilage defects will be defined as any increase in cartilage defect score in either tibial or femoral cartilage over 2 years.
Progression of BMLs BMLs will be defined as discrete subchondral areas with increased signal intensity and illdefined margins on sagittal proton density images, and graded at tibial and femoral sites (0-3). If multiple lesions are present at one site, the highest grade will be recorded. The intra- and inter-reader correlation coefficient for this method has been reported to be $0.88-0.93$ [13]. The progression of BMLs will be defined as any increase in their grade in either tibial or femoral site over 2 years. The maximum areal size will also be measured for each lesion. The lesion with the largest size will be used if more than one lesion is present at the same site. Our intra-observer correlation coefficient for this method is 0.97 [38]. The change in areal size of BMLs over 2 years will be assessed since it is more sensitive to change, with positive results seen over 6 months in a previous clinical trial [39].

Change in knee symptoms and function Knee pain, stiffness and function will be assessed using a selfadministered validated questionnaire, WOMAC (the Western Ontario and McMaster Universities Osteoarthritis Index) [40], at each study visit. It will be used to describe the population, and change in symptoms and function measured by change in WOMAC score will be a secondary outcome.

\section{Other measurements Anthropometry}

Height (stadiometer), weight (electric scale) and body mass index (height/weight ${ }^{2}$ ) will be measured at baseline and 2 years.

\section{Radiographic knee OA}

A weight-bearing anteroposterior radiograph of the study knee will be scored for osteophytes and joint space narrowing on a four-point scale (0-3) using the Altman atlas [33]. Our intra- and inter-observer reliability is 0.93

Table 3 Magnetic resonance imaging sequences and parameters at three study sites

\begin{tabular}{|c|c|c|c|}
\hline & Machine and coil & T1 sagittal & Proton density sagittal \\
\hline Melbourne & $\begin{array}{l}\text { 3.0 T whole body MR unit (Achieva, } \\
\text { Philips Medical Systems), using a } \\
\text { commercial 16-channel transmit- } \\
\text { receive knee coil }\end{array}$ & $\begin{array}{l}\text { T1-weighted fat suppressed 3D gradient recall } \\
\text { acquisition in the steady state; flip angle } 15 \\
\text { degrees; repetition time } 25.9 \text { msec; echo time } \\
9.2 \text { msec; field of view } 16 \mathrm{~cm} ; 320 \times 320 \text { matrix; } \\
\text { one acquisition; partition thickness } 0.5 \mathrm{~mm}\end{array}$ & $\begin{array}{l}\text { Proton density fat-saturated acquisition; flip } \\
\text { angle } 90 \text { degrees; repetition time } 3,817 \mathrm{msec} ; \\
\text { echo time } 25 \mathrm{msec} \text {; field of view } 16 \mathrm{~cm} ; 720 \times \\
720 \text { matrix; slice thickness } 2.5 \mathrm{~mm}\end{array}$ \\
\hline Hobart & $\begin{array}{l}\text { 1.5 T whole-body MR unit (GE-Signa; } \\
\text { GE Healthcare, Buckinghamshire, UK) } \\
\text { using a dedicated 8-channel knee coil }\end{array}$ & $\begin{array}{l}\text { T1-weighted fat-saturated 3D gradient-recalled } \\
\text { acquisition; flip angle } 30 \text { degrees; repetition } \\
\text { time } 31 \mathrm{msec} \text {; echo time } 6.8 \mathrm{msec} \text {; field of view } \\
16 \mathrm{~cm} ; 512 \times 512 \text { matrix; one excitation; slice } \\
\text { thickness } 1.5 \mathrm{~mm}\end{array}$ & $\begin{array}{l}\text { Proton density fat-saturated } 2 D \text { fast spin echo } \\
\text { sequence; flip angle } 150 \text { degrees; repetition } \\
\text { time } 3,800 \text { msec; echo time } 39 \text { msec; field of } \\
\text { view } 16 \mathrm{~cm} ; 512 \times 512 \text { matrix; } 3 \text { excitations; } \\
\text { slice thickness } 3 \mathrm{~mm}\end{array}$ \\
\hline Adelaide & $\begin{array}{l}1.5 \text { T whole-body MR unit (Aera, } \\
\text { Siemens) using a dedicated } \\
15 \text {-channel transmit-receive } \\
\text { knee coil }\end{array}$ & $\begin{array}{l}\text { T1-weighted fat-saturated 3D gradient-recalled } \\
\text { acquisition; flip angle } 30 \text { degrees, repetition } \\
\text { time } 14.7 \text { msec; echo time } 6.74 \text { msec; field of } \\
\text { view } 16 \mathrm{~cm} ; 448 \times 448 \text { matrix; one excitation; } \\
\text { slice thickness } 1.5 \mathrm{~mm}\end{array}$ & $\begin{array}{l}\text { Proton density fat-saturated } 2 D \text { fast spin echo } \\
\text { sequence; flip angle } 180 \text { degrees; repetition } \\
\text { time } 3,200 \text { msec; echo time } 39 \text { msec; field of } \\
\text { view } 16 \mathrm{~cm} ; 320 \times 320 \text { matrix; } 1 \text { excitation; slice } \\
\text { thickness } 3 \text { mm }\end{array}$ \\
\hline
\end{tabular}


and 0.86 for osteophytes and 0.93 and 0.85 for joint space narrowing, respectively [41].

\section{Knee angle}

The knee angle will be measured from weight-bearing radiographs, as it affects cartilage volume change [42]. Our intra-observer correlation coefficient is 0.98 [42].

\section{Tibial plateau bone area}

The cross-sectional area of tibial plateau will be measured manually from the reformatted axial MR images. The coefficient of variation of this method is $2.3-2.4 \%$ [34]. Cartilage volume will be adjusted for bone area to account for joint size.

\section{Meniscal tear and extrusion}

The presence of meniscal tears and extent of meniscal extrusion will be determined from sagittal images and confirmed in coronal and axial images [43], as they affect cartilage volume change [43]. The intra- and interreader correlation coefficient has been reported to be $0.85-0.96$ [43].

\section{Physical activity}

Physical activity will be measured using the International Physical Activity Questionnaire (IPAQ) [44] short version at baseline and 2 years.

\section{Lower limb muscle strength}

Lower limb muscle strength is expected to increase with a decrease in pain, and therefore will be measured by dynamometry at months 0,12 and 24 . The muscles measured in this technique are mainly quadriceps and hip flexors. The devices will be calibrated by suspending known weights at regular intervals. Repeatability estimates (Cronbach's) were 0.91 [45].

\section{Concomitant medication use}

Concomitant medication use including glucosamine, chondroitin, corticosteroids and non-steroidal antiinflammatory drugs will be allowed during the trial but will be documented. There is no definitive data showing that these treatments affect cartilage loss. The randomisation process is the most effective method for ensuring that two groups are as similar as possible with respect to known confounders and unknown potential confounders including treatments. We will adjust for medication use in the analyses.

\section{Cigarette smoking history}

Cigarette smoking history will be assessed at baseline and 2 years. As it affects cartilage loss and BMLs [46], we will adjust for smoking status in the analyses.

\section{Knee injury}

Knee injury will be assessed at baseline and 2 years. Prior knee injury is an exclusion criterion. Any significant injury during the study will be documented, as it is a risk factor for knee OA.

\section{Safety assessment}

Adverse events will be monitored throughout the study. Standard safety and efficacy monitoring will be performed through regular face-to-face visits and phone calls between visits. The participants are requested to report any adverse events to the research staff spontaneously. Biochemical testing (liver function tests, creatine kinase and renal function tests) will be performed at 4 weeks, and at 6, 12 and 24 months. Details of the adverse event and its relationship with study intervention will be recorded and reported to the Ethics Committees.

\section{Sample size calculation \\ Primary outcome}

Based on our previous published data, the rate of tibial cartilage volume loss in the control group was $3.0+/-3.0$ per annum [47]. A $33 \%$ reduction (that is, $1 \%$ ) in this rate is clinically significant since this indicates a significant reduction in structural progression of cartilage damage. Our previous work has shown that a $1 \%$ reduction in the annual rate of cartilage volume loss over 2 years decreased the need for knee replacement surgery by $20 \%$ over a 3-year period based on the strong association between the rate of cartilage volume loss and subsequent knee replacement [48]. With 140 participants in each arm, we will have $80 \%$ power to detect a $30 \%$ reduction in the rate of cartilage volume loss in the intervention group (2.0\%) compared with the control group (3.0\%), with alpha error 0.05 , two-sided significance. Given our previous experience in such studies, we expect a maximum dropout rate of $20 \%$ over 2 years. Therefore, a total of 350 participants (175 in each arm of the study) will be recruited.

\section{Secondary outcome}

Based on our reported progression rate of $68 \%$ for cartilage defects [49] and $21 \%$ [14] for BMLs over 2 years, with 140 participants per arm we will have $80 \%$ power to detect a $17 \%$ difference in the progression of cartilage defects and a $12 \%$ difference in the progression of BMLs between the intervention group and the control group. With 140 in each arm, we will be able to detect a $30 \%$ reduction in the WOMAC pain subscale in the intervention group.

\section{Statistical analysis}

The primary analyses will be intention-to-treat analyses of primary and secondary outcomes. Per protocol analyses (according to protocol adherence for patients who have taken all the study medication and have had all the 
outcome measures) will be performed as the secondary analyses. Intention-to-treat analysis will be carried out by using multiple imputation [50], provided that patients have had a baseline MRI. This analysis only requires missing data to be missing at random to be valid. Differences within a treatment group between follow-up and baseline measures will be assessed using a paired samples $t$-test. The clinical efficacy measures, that is, changes in pain and function, will also be analysed using the normalised area under the curve for difference in scores from baseline to month 24. Differences between treatment groups will be assessed using independent samples $t$-tests, ANCOVA (for continuous variables) or chi-squared tests (for dichotomous variables). Multiple linear regression for continuous endpoints or logistic regression for binary endpoints could be carried out as supplementary analyses for additional adjustment for imbalanced baseline factors. Analyses of treatment efficacy will be done by censoring participants at the time of any protocol deviation and developing a model for the probability of deviation, followed by weighted analyses using only the uncensored participants where the weights are the inverse probability of censoring. This produces estimates of treatment effect as if there were full compliance with the protocol in this trial and is far preferable to per protocol analyses based on (unweighted) observed compliance [51]. Pre-specified analyses to identify subgroups which may respond better to treatment will be examined using stratified analyses; variables include radiographic OA and co-pathology present on MRI.

\section{Data integrity and management}

All collected data are recorded using case report forms which will be processed centrally at the Clinical Informatics and Data Management Unit, Department of Epidemiology and Preventive Medicine, Monash University. The hard copies of the case report forms will be stored in a locked area at each study site with secured and restricted access. The electronic data will be stored in a password protected database with secured and restricted access. All data collected will be kept strictly confidential. Data transfer will be encrypted with all data de-identified.

\section{Withdrawal}

If participants withdraw from the study before 2 years of follow-up, the reason and date will be recorded. If the participant withdraws after a minimum of 6 months of treatment, he/she will be requested to have a second knee MRI scan and complete the questionnaires.

\section{Monitoring}

The principal investigators will monitor the conduct and progress of the project at each site. The trial coordinator will visit each study site to make sure that all trial procedures are compliant with the trial protocol. The principal investigators and the research team will have regular teleconferences to ensure efficient study execution and ongoing monitoring of the study progress, with summary documents circulated after each meeting. An independent Data and Safety Monitoring Board was convened, consisting of a clinical rheumatologist, a clinical cardiologist experienced in statin use, and a biostatistician, all with clinical trial experience. They will monitor adverse events. They will meet annually or more often if severe adverse events occur, and provide a written report to the principal investigators.

\section{Discussion}

We proposed a multicentre, randomised, double-blind, placebo-controlled trial to determine whether atorvastatin has a disease modifying effect in symptomatic knee OA by slowing the structural progression of knee OA and improving knee symptoms. If atorvastatin is proven to be effective, it will offer a novel therapeutic approach to reducing the progression of knee OA.

Oral administration of statins is an established, safe and well-tolerated treatment for the prevention of cardiovascular events [23]. Extending the potential use of statins to patients with knee OA is plausible. There is in vitro and in vivo evidence that statins may reduce the progression of OA via a number of pathways including their effects on lipid metabolism and inflammation. Statins reduce the levels of C-reactive protein and the production of inflammatory cytokines including IL- 6 and IL-1 $\beta$, most likely through their inhibition of NF-kB activation in monocytes or endothelial cells exposed to inflammatory stimuli [52]. Statins inhibit IL-1 $\beta$ induced production of matrix metalloproteinases (MMPs), and stimulate bone morphogenetic protein 2, aggrecan, and synthesis of type II collagen and cartilage matrix proteoglycan by chondrocytes [53], which is protective against cartilage damage. Atorvastatin inhibited IL-1beta-induced glycosaminoglycan release, TNF-alpha, MMP-13, and superoxide anion formation, protecting cartilage degradation following IL-1beta-stimulated cartilage in an in vitro OA model [54]. In animal models, statins reduce inflammatory cell infiltration and matrix-degrading enzyme expression and inhibit pro-inflammatory cytokines, thus reducing articular cartilage degeneration and the severity and progression of OA or arthritis [55-58]. In an anterior cruciate ligament transaction induced rabbit OA model, intraarticular statin injections reduced the gross morphological and histological changes in articular cartilage [55]. In a mouse model resembling human lipoprotein metabolism, atorvastatin significantly suppressed OA development [59]. In a rabbit model of early experimental OA, intra-articular application of atorvastatin 
showed chondroprotective effects both macroscopically and histopathologically [60].

The previous human studies [25-30] (Table 1) are all of an observational nature and thus susceptible to selection bias, information bias and confounding, and have used insensitive tools to assess disease progression. Most of these studies examined general OA without stratifying by different joints, since emerging evidence suggests different pathogenic mechanisms of OA in knee and hip joints, with the knee affected more by metabolic factors than the hip [7]. A randomised controlled trial is required to determine whether statin use affects the structural progression of knee OA.

Although OA is a disease of the whole joint, progressive articular cartilage loss is the hallmark of disease progression. Radiographic joint space narrowing is the gold standard to assess disease progression over time and has been used as the primary endpoint to examine the effect of disease modifying OA drugs in clinical trials. However, radiographic joint space narrowing provides a crude, insensitive method to assess disease progression [36]. MRI allows non-invasive direct visualisation of all joint components and direct measurement of articular cartilage, representing a sensitive method to assess OA progression. Cartilage volume has been validated by comparison with anatomical specimens [61]. It is a clinically useful measure which is inversely correlated with radiological grade of OA [41]. Cartilage volume loss is clinically significant, as it predicts important patient outcomes of pain [62] and risk of joint replacement [48]. Cartilage defects have been validated against surgically confirmed cartilage lesions and arthroscopy [63]. They are associated with knee pain [12], predict cartilage loss independent of cartilage volume [64], and predict joint replacement [49], thus providing a further assessment of cartilage health. Being the most common subchondral bone abnormality described in OA, BMLs are associated with knee pain [65], predict disease progression [66], cartilage volume loss [13] and joint replacement [14]. Thus, these MRIassessed knee structural changes have been chosen as the primary and secondary outcome measures in this study.

In summary, knee OA is the most common form of chronic joint disorder but has no proven pharmacological treatment that reduces structural disease progression. There is evidence that both inflammation and lipid metabolism contribute to the progression of OA. The anti-inflammatory and lipid lowering effects of stains make this class of drug a potential treatment approach to slowing the progression of OA. This randomised controlled trial will provide high-quality evidence to address whether atorvastatin has a novel disease modifying effect in OA by delaying the structural and symptomatic progression of knee OA. If demonstrated to be effective, atorvastatin could be used as a novel disease modifying agent to delay the onset of end-stage $\mathrm{OA}$ and the need for primary joint replacement for OA and possibly revision surgery. This has major public health importance, as it would produce substantial cost savings by delaying and possibly reducing the need for joint replacement surgery, and provide marked improvements in quality of life for people with knee OA.

\section{Trial status}

Upon submission, the OAKS study is in the process of patient recruitment.

\section{Abbreviations}

ACR: American College of Rheumatology; BML: bone marrow lesion; HDL: high-density lipoprotein; HMG-CoA: hydroxy-methyl-glutaryl coenzyme A; IL: interleukin; IPAQ: International Physical Activity Questionnaire; LDL: low-density lipoprotein; MMP: matrix metalloproteinase; MRI: magnetic resonance imaging; OA: osteoarthritis; OAKS: Osteoarthritis of the Knee Statin; TNF: tumour necrosis factor; VAS: visual analogue scale; WOMAC: Western Ontario and McMaster Universities Osteoarthritis Index.

\section{Competing interests}

The authors declare that they have no competing interests.

\section{Authors' contributions}

YW and FMC conceived the study; YW, FMC, AT, GJ, CH, CD, AEW and AF were all involved in study design and execution. YW and FMC drafted the manuscript. All authors revised the manuscript and gave final approval of the version to be submitted.

\section{Acknowledgements}

Ms Judy Hankin, Ms Alice Noone, Ms Molly Bond, Mr Cameron Redpath, Ms Clare Bellhouse, Dr Andrew Teichtahl, Dr Sharmayne Brady, Dr Louisa Chou, Dr Dawn Aitken, Ms Lizzy Reid, Ms Sarah Day, Ms Sarah Downie-Doyle and Ms Carlee Schultz have been involved in the coordination and execution of this study.

\section{Funding}

The OAKS study is supported by the National Health and Medical Research Council of Australia (NHMRC, ID 1048581). YW is the recipient of an NHMRC Career Development Fellowship (Clinical Level 1 number 1065464). GJ is the recipient of an NHMRC Practitioner Fellowship (1023222). CD is the recipient of an Australia Research Council Future Fellowship. AEW is the recipient of an NHMRC Career Development Fellowship (Clinical Level 2 number 1063574).

\section{Author details}

'Department of Epidemiology and Preventive Medicine, Monash University, Alfred Hospital, Melbourne 3004, Australia. ${ }^{2}$ Menzies Institute for Medical Research, University of Tasmania, Private Bag 23, Hobart, TAS 7000, Australia. ${ }^{3}$ The Queen Elizabeth Hospital, University of Adelaide, Woodville, SA 5011, Australia. ${ }^{4}$ Discipline of Medicine, University of Adelaide, Adelaide, SA 5005, Australia.

Received: 28 June 2015 Accepted: 14 December 2015

Published online: 23 December 2015

\section{References}

1. Vos T, Flaxman AD, Naghavi M, Lozano R, Michaud C, Ezzati M, et al. Years lived with disability (YLDs) for 1160 sequelae of 289 diseases and injuries 1990-2010: a systematic analysis for the Global Burden of Disease Study 2010. Lancet (London, England). 2012;380:2163-96.

2. Murphy L, Schwartz TA, Helmick CG, Renner JB, Tudor G, Koch G, et al. Lifetime risk of symptomatic knee osteoarthritis. Arthritis Rheum. 2008;59: 1207-13.

3. Qvist P, Bay-Jensen AC, Christiansen C, Dam EB, Pastoureau P, Karsdal MA. The disease modifying osteoarthritis drug (DMOAD): is it in the horizon? Pharmacol Res. 2008;58:1-7. 
4. Velasquez MT, Katz JD. Osteoarthritis: another component of metabolic syndrome? Metab Syndr Relat Disord. 2010;8:295-305.

5. Masuko K, Murata M, Suematsu N, Okamoto K, Yudoh K, Nakamura H, et al. A metabolic aspect of osteoarthritis: lipid as a possible contributor to the pathogenesis of cartilage degradation. Clin Exp Rheumatol. 2009;27:347-53.

6. Goldring MB, Otero M. Inflammation in osteoarthritis. Curr Opin Rheumatol. 2011;23:471-8

7. Wang Y, Simpson JA, Wluka AE, Teichtahl AJ, English DR, Giles GG, et al. Relationship between body adiposity measures and risk of primary knee and hip replacement for osteoarthritis: a prospective cohort study. Arthritis Res Ther. 2009;11:R31.

8. Hart DJ, Doyle DV, Spector TD. Association between metabolic factors and knee osteoarthritis in women: the Chingford Study. J Rheumatol. 1995;22:1118-23.

9. Sturmer T, Sun Y, Sauerland S, Zeissig I, Gunther KP, Puhl W, et al. Serum cholesterol and osteoarthritis. The baseline examination of the UIm Osteoarthritis Study. J Rheumatol. 1998;25:1827-32.

10. Davies-Tuck ML, Hanna F, Davis SR, Bell RJ, Davison SL, Wluka AE, et al. Total cholesterol and triglycerides are associated with the development of new bone marrow lesions in asymptomatic middle-aged women - a prospective cohort study. Arthritis Res Ther. 2009;11:R181.

11. Dore D, de Hoog J, Giles G, Ding C, Cicuttini F, Jones G. A longitudinal study of the association between dietary factors, serum lipids and bone marrow lesions of the knee. Arthritis Res Ther. 2012;14:R13.

12. Zhai G, Blizzard L, Srikanth V, Ding C, Cooley H, Cicuttini F, et al. Correlates of knee pain in older adults: Tasmanian Older Adult Cohort Study. Arthritis Rheum. 2006;55:264-71.

13. Raynauld JP, Martel-Pelletier J, Berthiaume MJ, Abram F, Choquette D, Haraoui B, et al. Correlation between bone lesion changes and cartilage volume loss in patients with osteoarthritis of the knee as assessed by quantitative magnetic resonance imaging over a 24-month period. Ann Rheum Dis. 2008;67:683-8.

14. Tanamas SK, Wluka AE, Pelletier JP, Pelletier JM, Abram F, Berry PA, et al. Bone marrow lesions in people with knee osteoarthritis predict progression of disease and joint replacement: a longitudinal study. Rheumatology (Oxford). 2010;49:2413-9.

15. Smith MD, Triantafillou S, Parker A, Youssef PP, Coleman M. Synovia membrane inflammation and cytokine production in patients with early osteoarthritis. J Rheumatol. 1997;24:365-71.

16. Ayral X, Pickering EH, Woodworth TG, Mackillop N, Dougados M. Synovitis: a potential predictive factor of structural progression of medial tibiofemoral knee osteoarthritis - results of a 1 year longitudinal arthroscopic study in 422 patients. Osteoarthritis Cartilage. 2005;13:361-7.

17. Roemer FW, Zhang Y, Niu J, Lynch JA, Crema MD, Marra MD, et al. Tibiofemoral joint osteoarthritis: risk factors for MR-depicted fast cartilage loss over a 30-month period in the multicenter osteoarthritis study. Radiology. 2009;252:772-80.

18. Hanna FS, Bell RJ, Cicuttini FM, Davison SL, Wluka AE, Davis SR. High sensitivity C-reactive protein is associated with lower tibial cartilage volume but not lower patella cartilage volume in healthy women at mid-life. Arthritis Res Ther. 2008;10:R27.

19. Spector TD, Hart DJ, Nandra D, Doyle DV, Mackillop N, Gallimore JR, et al. Lowlevel increases in serum C-reactive protein are present in early osteoarthritis of the knee and predict progressive disease. Arthritis Rheum. 1997:40:723-7.

20. Livshits G, Zhai G, Hart DJ, Kato BS, Wang H, Williams FM, et al. Interleukin-6 is a significant predictor of radiographic knee osteoarthritis: The Chingford study. Arthritis Rheum. 2009;60:2037-45.

21. Pelletier JP, Raynauld JP, Caron J, Mineau F, Abram F, Dorais M, et al. Decrease in serum level of matrix metalloproteinases is predictive of the disease-modifying effect of osteoarthritis drugs assessed by quantitative MRI in patients with knee osteoarthritis. Ann Rheum Dis. 2010;69:2095-101.

22. Stannus $\mathrm{O}$, Jones G, Cicuttini F, Parameswaran V, Quinn S, Burgess J, et al. Circulating levels of IL-6 and TNF-alpha are associated with knee radiographic osteoarthritis and knee cartilage loss in older adults. Osteoarthritis Cartilage. 2010;18:1441-7.

23. Baigent C, Blackwell L, Emberson J, Holland LE, Reith C, Bhala N, et al. Efficacy and safety of more intensive lowering of LDL cholesterol: a meta-analysis of data from 170,000 participants in 26 randomised trials. Lancet (London, England). 2010;376:1670-81

24. Lazzerini PE, Capecchi PL, Selvi E, Lorenzini S, Bisogno S, Baldari CT, et al. Statins and the joint: multiple targets for a global protection? Semin Arthritis Rheum. 2011;40:430-46.
25. Clockaerts S, Van Osch GJ, Bastiaansen-Jenniskens YM, Verhaar JA, Van Glabbeek F, Van Meurs JB, et al. Statin use is associated with reduced incidence and progression of knee osteoarthritis in the Rotterdam study. Ann Rheum Dis. 2012;71:642-7.

26. Chodick G, Amital H, Shalem Y, Kokia E, Heymann AD, Porath A, et al. Persistence with statins and onset of rheumatoid arthritis: a populationbased cohort study. PLoS Med. 2010;7:e1000336.

27. Kadam UT, Blagojevic M, Belcher J. Statin use and clinical osteoarthritis in the general population: a longitudinal study. J Gen Intern Med. 2013;28:943-9.

28. Mansi IA, Mortensen EM, Pugh MJ, Wegner M, Frei CR. Incidence of musculoskeletal and neoplastic diseases in patients on statin therapy: results of a retrospective cohort analysis. Am J Med Sci. 2013;345:343-8.

29. Beattie MS, Lane NE, Hung YY, Nevitt MC. Association of statin use and development and progression of hip osteoarthritis in elderly women. J Rheumatol. 2005:32:106-10.

30. Riddle DL, Moxley G, Dumenci L. Associations between statin use and changes in pain, function and structural progression: a longitudinal study of persons with knee osteoarthritis. Ann Rheum Dis. 2013;72:196-203.

31. Schulz KF, Altman DG, Moher D. CONSORT 2010 statement: updated guidelines for reporting parallel group randomised trials. BMJ (Clinical research ed). 2010;340:c332.

32. Altman R, Asch E, Bloch D, Bole G, Borenstein D, Brandt $K$, et al. Development of criteria for the classification and reporting of osteoarthritis. Classification of osteoarthritis of the knee. Diagnostic and Therapeutic Criteria Committee of the American Rheumatism Association. Arthritis Rheum. 1986;29:1039-49.

33. Altman RD, Hochberg M, Murphy Jr WA, Wolfe F, Lequesne M, Suppl A. Atlas of individual radiographic features in osteoarthritis. Osteoarthritis Cartilage. 1995;3(Suppl A):3-70.

34. Wluka AE, Stuckey S, Brand C, Cicuttini FM. Supplementary vitamin E does not affect the loss of cartilage volume in knee osteoarthritis: a 2 year double blind randomized placebo controlled study. J Rheumatol. 2002;29: 2585-91.

35. Wang Y, Hall S, Hanna F, Wluka AE, Grant G, Marks P, et al. Effects of Hylan G-F 20 supplementation on cartilage preservation detected by magnetic resonance imaging in osteoarthritis of the knee: a two-year single-blind clinical trial. BMC Musculoskelet Disord. 2011;12:195.

36. Raynauld JP, Martel-Pelletier J, Bias P, Laufer S, Haraoui B, Choquette D, et al. Protective effects of licofelone, a 5-lipoxygenase and cyclo-oxygenase inhibitor, versus naproxen on cartilage loss in knee osteoarthritis: a first multicentre clinical trial using quantitative MRI. Ann Rheum Dis. 2009;68:938-47.

37. Ding C, Garnero P, Cicuttini F, Scott F, Cooley H, Jones G. Knee cartilage defects: association with early radiographic osteoarthritis, decreased cartilage volume, increased joint surface area and type II collagen breakdown. Osteoarthritis Cartilage. 2005;13:198-205.

38. Dore D, Quinn S, Ding C, Winzenberg T, Zhai G, Cicuttini F, et al. Natural history and clinical significance of MRI-detected bone marrow lesions at the knee: a prospective study in community dwelling older adults. Arthritis Res Ther. 2010;12:R223.

39. Laslett LL, Dore DA, Quinn SJ, Boon P, Ryan E, Winzenberg TM, et al. Zoledronic acid reduces knee pain and bone marrow lesions over 1 year: a randomised controlled trial. Ann Rheum Dis. 2012;71:1322-8.

40. Bellamy N, Buchanan WW, Goldsmith CH, Campbell J, Stitt LW. Validation study of WOMAC: a health status instrument for measuring clinically important patient relevant outcomes to antirheumatic drug therapy in patients with osteoarthritis of the hip or knee. J Rheumatol. 1988;15:1833-40.

41. Cicuttini FM, Wluka AE, Forbes A, Wolfe R. Comparison of tibial cartilage volume and radiologic grade of the tibiofemoral joint. Arthritis Rheum. 2003:48:682-8.

42. Cicuttini F, Wluka A, Hankin J, Wang Y. Longitudinal study of the relationship between knee angle and tibiofemoral cartilage volume in subjects with knee osteoarthritis. Rheumatology (Oxford). 2004;43:321-4.

43. Raynauld JP, Martel-Pelletier J, Berthiaume MJ, Beaudoin G, Choquette D, Haraoui $B$, et al. Long term evaluation of disease progression through the quantitative magnetic resonance imaging of symptomatic knee osteoarthritis patients: correlation with clinical symptoms and radiographic changes. Arthritis Res Ther. 2006;8:R21.

44. Craig CL, Marshall AL, Sjostrom M, Bauman AE, Booth ML, Ainsworth BE, et al. International physical activity questionnaire: 12-country reliability and validity. Med Sci Sports Exerc. 2003;35:1381-95. 
45. Jones G, Glisson M, Hynes K, Cicuttini F. Sex and site differences in cartilage development: a possible explanation for variations in knee osteoarthritis in later life. Arthritis Rheum. 2000;43:2543-9.

46. Davies-Tuck ML, Wluka AE, Forbes A, Wang Y, English DR, Giles GG, et al. Smoking is associated with increased cartilage loss and persistence of bone marrow lesions over 2 years in community-based individuals. Rheumatology (Oxford). 2009;48:1227-31.

47. Bennell KL, Bowles KA, Wang Y, Cicuttini F, Davies-Tuck M, Hinman RS. Higher dynamic medial knee load predicts greater cartilage loss over 12 months in medial knee osteoarthritis. Ann Rheum Dis. 2011;70:1770-4.

48. Cicuttini FM, Jones G, Forbes A, Wluka AE. Rate of cartilage loss at two years predicts subsequent total knee arthroplasty: a prospective study. Ann Rheum Dis. 2004;63:1124-7.

49. Wluka AE, Ding C, Jones G, Cicuttini FM. The clinical correlates of articular cartilage defects in symptomatic knee osteoarthritis: a prospective study. Rheumatology (Oxford). 2005;44:1311-6.

50. Little RJ, D'Agostino R, Cohen ML, Dickersin K, Emerson SS, Farrar JT, et al. The prevention and treatment of missing data in clinical trials. N Engl J Med. 2012; 367:1355-60.

51. Hernan MA, Lanoy E, Costagliola D, Robins JM. Comparison of dynamic treatment regimes via inverse probability weighting. Basic Clin Pharmacol Toxicol. 2006:98:237-42.

52. Baker JF, Walsh P, Mulhall KJ. Statins: a potential role in the management of osteoarthritis? Joint Bone Spine. 2011;78:31-4.

53. Yudoh K, Karasawa R. Statin prevents chondrocyte aging and degeneration of articular cartilage in osteoarthritis (OA). Aging (Albany NY). 2010;2:990-8.

54. Pathak NN, Lingaraju MC, Balaganur V, Kant V, More AS, Kumar D, et al. Anti-inflammatory and chondroprotective effects of atorvastatin in a cartilage explant model of osteoarthritis. Inflamm Res. 2015;64:161-9.

55. Akasaki Y, Matsuda S, Nakayama K, Fukagawa S, Miura H, Iwamoto Y. Mevastatin reduces cartilage degradation in rabbit experimental osteoarthritis through inhibition of synovial inflammation. Osteoarthritis Cartilage. 2009;17:235-43.

56. Barsante MM, Roffe E, Yokoro CM, Tafuri WL, Souza DG, Pinho V, et al. Anti-inflammatory and analgesic effects of atorvastatin in a rat model of adjuvant-induced arthritis. Eur J Pharmacol. 2005;516:282-9.

57. Leung BP, Sattar N, Crilly A, Prach M, McCarey DW, Payne H, et al. A novel anti-inflammatory role for simvastatin in inflammatory arthritis. J Immunol. 2003;170:1524-30.

58. Aktas E, Sener E, Gocun PU. Mechanically induced experimental knee osteoarthritis benefits from anti-inflammatory and immunomodulatory properties of simvastatin via inhibition of matrix metalloproteinase-3. J Orthop Traumatol. 2011;12:145-51.

59. Gierman LM, Kuhnast S, Koudijs A, Pieterman EJ, Kloppenburg M, van Osch GJ, et al. Osteoarthritis development is induced by increased dietary cholesterol and can be inhibited by atorvastatin in APOE*3Leiden.CETP mice - a translational model for atherosclerosis. Ann Rheum Dis. 2014;73:921-7.

60. Dinc M, Bilgen MS, Kucukalp A, Bilgen OF. An assessment of the chondroprotective effects of intra-articular application of statin and tetracycline on early-stage experimental osteoarthritis. ISRN Orthop. 2012; 2012:182097.

61. Cicuttini F, Forbes A, Morris K, Darling S, Bailey M, Stuckey S. Gender differences in knee cartilage volume as measured by magnetic resonance imaging. Osteoarthritis Cartilage. 1999;7:265-71.

62. Wluka AE, Wolfe R, Stuckey S, Cicuttini FM. How does tibial cartilage volume relate to symptoms in subjects with knee osteoarthritis? Ann Rheum Dis. 2004;63:264-8.

63. Disler DG, McCauley TR, Wirth CR, Fuchs MD. Detection of knee hyaline cartilage defects using fat-suppressed three-dimensional spoiled gradientecho MR imaging: comparison with standard MR imaging and correlation with arthroscopy. AJR Am J Roentgenol. 1995;165:377-82.

64. Cicuttini F, Ding C, Wluka A, Davis S, Ebeling PR, Jones G. Association of cartilage defects with loss of knee cartilage in healthy, middle-age adults: A prospective study. Arthritis Rheum. 2005;52:2033-9.

65. Felson DT, Chaisson CE, Hill CL, Totterman SM, Gale ME, Skinner KM, et al. The association of bone marrow lesions with pain in knee osteoarthritis. Ann Intern Med. 2001;134:541-9.

66. Felson DT, McLaughlin S, Goggins J, LaValley MP, Gale ME, Totterman S, et al. Bone marrow edema and its relation to progression of knee osteoarthritis. Ann Intern Med. 2003;139:330-6.

\section{Submit your next manuscript to BioMed Central and we will help you at every step:}

- We accept pre-submission inquiries

- Our selector tool helps you to find the most relevant journal

- We provide round the clock customer support

- Convenient online submission

- Thorough peer review

- Inclusion in PubMed and all major indexing services

- Maximum visibility for your research

Submit your manuscript at www.biomedcentral.com/submit
Biomed Central 